\title{
Searches at the Run II Tevatron Collider
}

\author{
Leslie S. Groer \\ for the $C D F$ and $D \varnothing$ Collaborations. \\ Department of Physics, Columbia University, New York, New York 10027, USA. \\ E-mail: groer@fnal.gov
}

\begin{abstract}
.
Some initial results based on $40-90 \mathrm{pb}^{-1}$ of integrated luminosity from Run II are presented for searches for new phenomena beyond the Standard Model, conducted at the upgraded Tevatron CDF and $\mathrm{D} \emptyset$ experiments.
\end{abstract}

Run II commenced at the upgraded Tevatron proton-antiproton collider complex in 2001 with a center-of-mass collision energy of $1.96 \mathrm{TeV}$. Both the CDF and DØ detectors underwent major upgrades to take full advantage of the increased instantaneous luminosity, with the installation of new inner tracking systems within solenoidal fields, including silicon vertex detectors, upgrades and extensions to the muon detection systems and new electronics, trigger and data acquisition systems for the higher rate with the reduced bunch crossing time of $396 \mathrm{~ns}$. The Tevatron is delivering approximately $7 \mathrm{pb}^{-1}$ a week with a peak instantaneous luminosity achieved of $4.5 \times 10^{31} \mathrm{~cm}^{-2} \mathrm{~s}^{-1}$ and has delivered a total of $240 \mathrm{pb}^{-1}$.

The general purpose detectors with very good azimuthal and forward coverage allow the identification of many final states that could provide hints of new physics. Some of these searches, from approximately $40-90 \mathrm{pb}^{-1}$ of integrated luminosity that has been analyzed, are presented below.

\section{Searches for Higgs Bosons}

One of the main interests in Run II is the search for Higgs bosons, either from the Standard Model (SM) or one of the many varieties predicted by extensions to the SM. The predicted inclusive production cross section for a SM Higgs boson at $1.96 \mathrm{TeV}$ is of order $1 \mathrm{pb}$, with gluon-gluon fusion being the dominant process. For a SM Higgs mass less than $135 \mathrm{GeV} / \mathrm{c}^{2}$ the dominant decay is to $b \bar{b}$ quark pairs. Searches in this channel however are swamped by the large production cross section of $b$-quark pairs from QCD jet production; a more fruitful search is for the production of a Higgs boson in association with a heavy vector boson $\left(p \bar{p} \rightarrow W^{ \pm *} / Z^{0 *} \rightarrow W^{ \pm} H^{0} / Z^{0} H^{0}\right)$, where the vector boson decays leptonically. For SM Higgs masses greater than $135 \mathrm{GeV} / \mathrm{c}^{2}$, the Higgs boson is predicted to decay predominantly to $W^{+} W^{-}$pairs. Again the leptonic decay channels yield the cleanest signatures.

Studies in the Tevatron Supersymmetry and Higgs Working Groups[1] indicated that a lower mass SM Higgs may be within the reach of the Tevatron Run II program provided 
sufficient luminosity can be delivered $\left(\sim 5-10 \mathrm{fb}^{-1}\right)$ and by combining all the decay channels from both experiments.

Both CDF and DØ have preliminary investigations in the associated production channels looking for either electron and muon pairs in the case of the $Z$ or a high- $\mathrm{p}_{T}$ electron or muon and a large imbalance in the transverse momentum (missing $\mathrm{E}_{T}$ ) in the case of a $W$. These channels are not yet competitive for setting limits but do prove the capabilities of the detectors and the analysis techniques.

$\mathrm{D} \varnothing$ has also searched for higgs decays to $W W$ pairs in the leptonic decay modes and sees zero events in the $e e$ channel in $44 \mathrm{pb}^{-1}$, and one event each in the $e \mu\left(34 \mathrm{pb}^{-1}\right)$ and $\mu \mu\left(48 \mathrm{pb}^{-1}\right)$ channels with expected backgrounds of $0.7 \pm 1.4,0.9 \pm 1.5$ and $0.3 \pm 0.1$ events respectively. These searches exploit the fact that the Higgs boson is a fundamental spin-zero scalar and hence angular spin correlations can be used to reduce the backgrounds from fakes and diboson and $t \bar{t}$ production. Combining these results sets an upper limit on the production of a Higgs boson times branching ratio to $W W$ pairs of $3 \mathrm{pb}$ at the $95 \%$ confidence level (CL), about two orders of magnitude above the SM predictions, but only a factor of ten above the predictions of more exotic models such as a fermiophobic or topcolor Higgs. Searches for Higgs decaying to pairs of high- $\mathrm{p}_{T}$ photons also set limits on the production times branching ratio of about $10-1 \mathrm{pb}$ for Higgs masses running from 60 to $120 \mathrm{GeV} / \mathrm{c}^{2}$. For Higgs masses of about $120 \mathrm{GeV} / \mathrm{c}^{2}$, the cross section limits are comparable to the theoretical expectations if $B(h \rightarrow \gamma \gamma)=1$.

CDF has searched for signatures of decays of doubly-charged higgs $\left(\mathrm{H}^{++}\right)$which are predicted in various classes of left-right symmetry breaking models [2]. These decays provide strong experimental signatures as there are very low backgrounds for same-sign high- $\mathrm{p}_{T}$ leptons in hadron collisions. Zero same-sign central electron events are observed

in $91 \pm 5.3 \mathrm{pb}^{-1}$, based on an inclusive electron trigger, with a signal acceptance of about $20-35 \%$ and an expected background of $0.6 \pm 0.5$ coming mainly from $Z, Q C D$ and $W+$ jets. The production cross section limit established at $95 \% \mathrm{CL}$ using a sliding mass window of $\mathrm{M}_{H^{++}} \pm 10 \%$ is $125-90 \mathrm{pb}$ for a $\mathrm{H}^{++}$mass going from 105 to $145 \mathrm{GeV} / \mathrm{c}^{2}$.

\section{Searches for Heavy Neutral Gauge Bosons and Extra Dimensions}

Both CDF and DØ have searched for evidence of new heavy neutral gauge bosons assuming SM couplings to both dielectron and dimuon lepton pairs. Neither experiment observes any excess with backgrounds dominated by Drell-Yan lepton pair-production and smaller contributions from misidentified electrons and diboson and $t \bar{t}$-pair production. The Run I limits were 690 and $670 \mathrm{GeV} / \mathrm{c}^{2}$ from CDF and D $\varnothing$ respectively. The current limits are $650(e e)$ and $455(\mu \mu) \mathrm{GeV} / \mathrm{c}^{2}$ from CDF in $72 \mathrm{pb}^{-1}$ giving a combined limit of $665 \mathrm{GeV} / \mathrm{c}^{2}$. The limit from DØ in the dielectron channel in $50 \mathrm{pb}^{-1}$ of integrated luminosity is $620 \mathrm{GeV} / \mathrm{c}^{2}$. The Run II sensitivity, which is enhanced by the increased cross sections at the higher center of mass energies (1.8 TeV in Run I), is approaching that of Run I.

Similar experimental signatures are indicated in the recently developed extra dimension models. DØ has searched for Large Extra Dimensions (LED) in the ADD [3] model assuming SM particles are confined to a 3-brane, but gravity propagates in the extra di- 
mensions which, if large, lead to an effective Planck scale $M_{S}$ in the TeV range. The expected signatures would be excesses of high-mass dilepton or diphoton pairs coupling to the the excited states of the graviton, the so-called Kaluza-Klein modes. DØ has searched in $50 \mathrm{pb}^{-1}$ of well-balanced high- $\mathrm{p}_{T}$ di“"em" events where dielectron and diphoton events are treated identically (i.e. with no track requirements) and in $30 \mathrm{pb}^{-1}$ of dimuon events. The data distributions are fitted to a signal plus background hypothesis to evaluate upper limits on the coupling $\eta_{G}$ which in turn can be translated into 95\% CL lower limits on $M_{S}$ in various formalisms. The limits on $M_{S}$ are of order $1 \mathrm{TeV}$ in the diem channel, very competitive with the Run I results. The new collider limit from the dimuon channel is of order $0.7 \mathrm{TeV}$. The Tevatron is expected to probe up to 1.6 (2) $\mathrm{TeV}$ with 0.3 (2) $\mathrm{fb}^{-1}$ in Run II.

CDF has also searched for anomalous dilepton production at large mass within the context of Randall-Sundrum (RS) graviton models which predict observable discrete spin-2 resonances [4]. The Kaluza Klein excitations of the graviton can be separately produced as resonances, enhancing the Drell-Yan cross section at large mass. CDF has compared the Drell Yan spectrum in the ee and $\mu \mu$ channel to the SM expectations in $72 \mathrm{pb}^{-1}$ and no excess has been observed. A 95\% CL upper limit on the cross section as a function of the RS graviton mass can be derived, which is $535 \mathrm{GeV} / \mathrm{c}^{2}$ in the dielectron and $370 \mathrm{GeV} / \mathrm{c}^{2}$ in the dimuon channel, giving a combined limit of $555 \mathrm{GeV} / \mathrm{c}^{2}$.

\section{Searches in Diphoton Events}

In gauge mediated supersymmetry the lightest supersymmetric particle (LSP) is a very light gravitino $(\widetilde{G})$ so the phenomenology is driven by the nature of the next to lightest supersymmetric particle (NLSP), the neutralino, which decays as $\bar{\chi}_{1}^{0} \rightarrow \gamma \widetilde{G} \rightarrow \gamma \gamma \widetilde{G}$. D $\emptyset$ has searched in this context for diphotons with $\mathrm{p}_{T}>20 \mathrm{GeV} / \mathrm{c}^{2}$ and large missing $\mathrm{E}_{T}$. No events are observed in $50 \mathrm{pb}^{-1}$ with expected backgrounds of $1.6 \pm 0.4$ coming from QCD fakes. A 95\% CL limit of $66 \mathrm{GeV} / \mathrm{c}^{2}$ for the mass of the NLSP is derived in the Snowmass model where $\mathrm{M}=2 \Lambda, N_{5}=1, \tan \beta=15$ and $\mu=0$ [5]. CDF has also not seen any anomalous high- $\mathrm{p}_{T}$ diphoton plus missing $\mathrm{E}_{T}$ events in $84 \mathrm{pb}^{-1}$.

\section{Searches in Dilepton and Trilepton Events}

Both CDF and DØ conduct model-independent searches for anomalous production of high- $\mathrm{p}_{T}$ dilepton and trilepton events with large missing $\mathrm{E}_{T}$, especially of different lepton flavors. These channels have very low backgrounds, dominated by $Z \rightarrow \tau \tau$ at low missing $\mathrm{E}_{T}$ and heavy vector-boson production with fake leptons and with smaller contributions from diboson and $t \bar{t}$ production at high missing $\mathrm{E}_{T}$. $\mathrm{D} \varnothing$ sees 13 events in the $e \mu$ channel in $33 \mathrm{pb}^{-1}$ with expected backgrounds of $9.6 \pm 2.7$ and sets $95 \%$ CL limits of acceptance times branching ratio for new physics of $0.4-0.1 \mathrm{pb}$ with missing $\mathrm{E}_{T}$

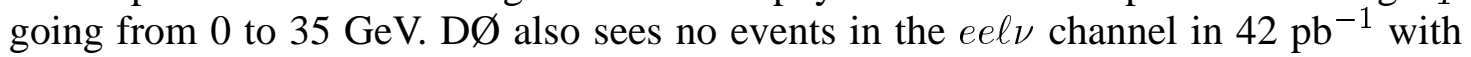
$0 \pm 2$ events expected from mostly heavy vector boson backgrounds and sets a 95\% CL limit on the production cross section times branching ratio of $3.5 \mathrm{pb}$. 


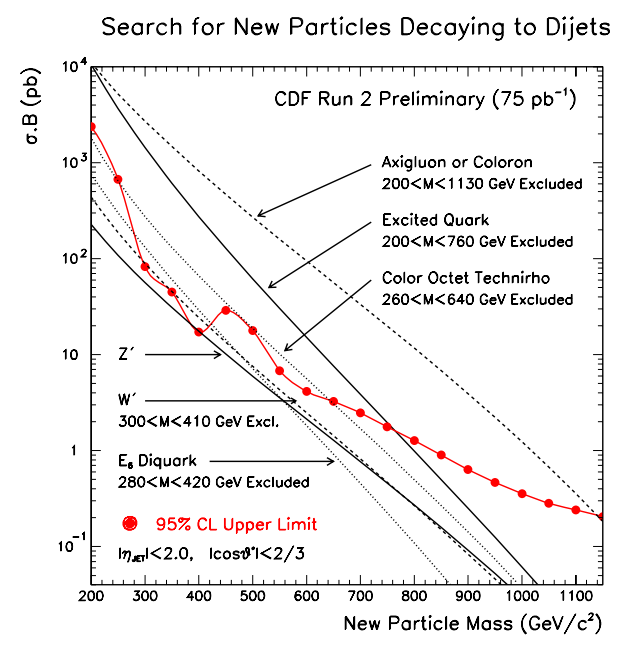

FIGURE 1. Preliminary CDF 95\% CL upper limits on $\sigma \cdot B$ for narrow dijet resonances for a variety of non-Standard Model models.

\section{Searches in Dijet Events}

The dijet mass spectrum has been investigated at both experiments as a test of QCD and for new mass resonances. CDF fits the mass spectrum in $75 \mathrm{pb}^{-1}$ with a simple background parameterization and searches for bumps comparable to the mass resolution. No significant evidence of any new resonance is found . Several 95\% CL upper limits can be derived (shown in Figure 1) for the cross section times branching ratio for a host of non-SM models which predict narrow dijet resonances: axigluons, flavor universal colorons, excited quarks, color octet technirhos, E6 diquarks and $W^{\prime}$ and $Z^{\prime}$ bosons. All these limits surpass the Run I results.

\section{Searches for Leptoquarks}

Extended gauge sector and composite models imply a direct coupling between the lepton and quark sectors via scalar leptoquarks (LQ) that can be pair produced from quark or gluon fusion. Models are characterized by the coupling $\beta=B(\mathrm{LQ} \rightarrow \ell q)=1-$ $B(\mathrm{LQ} \rightarrow \nu q)$. Strategies involve searching for dilepton and jets events and reconstructing the LQ mass or searching for events with missing $\mathrm{E}_{T}$ and dijets. CDF and DØ have seen no evidence for first $(e)$ or second $(\mu)$ generation leptoquarks with 95\% CL limits from $\mathrm{CDF}$ of $230 \mathrm{GeV} / \mathrm{c}^{2}$ for $\mathrm{LQ}_{1}$ from $72 \mathrm{pb}^{-1}$, an improvement over the $220 \mathrm{GeV} / \mathrm{c}^{2}$ limit from Run I. DØ's result for $\mathrm{LQ}_{2}$ is $157 \mathrm{GeV} / \mathrm{c}^{2}$ from $40 \mathrm{pb}^{-1}$, not yet competitive with the Run I result of $200 \mathrm{GeV} / \mathrm{c}^{2}$. CDF also conducts a flavor-blind search for $\mathrm{LQ} \rightarrow \nu q$ in $76 \mathrm{pb}^{-1}$ with limits of $M_{L Q}<60$ or $M_{L Q}>107 \mathrm{GeV} / \mathrm{c}^{2}$. 


\section{Search for Excited Electrons}

CDF has searched its ee $\gamma$ data for evidence of the decay of an excited or exotic electron $\left(p \bar{p} \rightarrow e^{*}+e \rightarrow e \gamma+e\right)$ which would indicate compositeness. No events are observed in $72 \mathrm{pb}^{-1}$ with expected background contributions coming mainly from $Z \gamma$ events and smaller contributions from $Z+$ jet, multijet and $W+$ jet, so a new mass limit can be set of $M_{e^{*}}>785 \mathrm{GeV} / \mathrm{c}^{2}$ at $95 \% \mathrm{CL}$, assuming a contact interaction model [6] and the compositeness scale $\Lambda=M_{e^{*}}$. This limit exceeds the previous published limit from $\mathrm{H} 1$ of $223 \mathrm{GeV} / \mathrm{c}^{2}$ [7].

\section{Charged Massive Stable Particles}

CDF has performed a search for charged massive stable particles that escape the detector without decaying and hence present themselves as slow-moving high- $\mathrm{p}_{T}$ muonlike objects. The time-of-flight detector (TOF) encasing the tracking volume at $140 \mathrm{~cm}$ from the beampipe with time resolution of 100 ps allows detection of these long-lived particles. In $53 \mathrm{pb}^{-1}$ of data, seven events are observed with $\Delta_{T O F}>2.5 \mathrm{~ns}$ and with $2.9 \pm 0.7_{\text {stat }} \pm 3.1_{\text {sys }}$ events expected.

The results can be interpreted in the context of several SUSY-based models (e.g. gauge-mediated supersymmetry breaking) where the LSP is a nearly massless Goldstino and the stop or stau is the NLSP, which can be long-lived, depending on the supersymmetry breaking scale. Interpreting the results assuming the stop as the stable NLSP gives a lower limit on the stop mass of $107 \mathrm{GeV} / \mathrm{c}^{2}$ for an isolated decay or $96 \mathrm{GeV} / \mathrm{c}^{2}$ for a stop decay associated with a jet. The previous limit on these models is from ALEPH at $95 \mathrm{GeV} / \mathrm{c}^{2}[8]$.

\section{CONCLUSION}

The Tevatron Run II is progressing steadily with very well performing detectors. Many of the results are already competitive or surpass those from Run I due to improved detectors and the higher center of mass collision energy. Sensitivity will increase at the $0.1 \mathrm{pb}$ and $1 \mathrm{TeV}$ scale in many channels as the integrated luminosity accumulates with the exciting potential for discoveries.

\section{REFERENCES}

1. Carena, M., et al., hep-ph/0010338(2000).

2. Gunion, J. F., Loomis, C., and Pitts, K. T., hep-ph/9610237 (1996).

3. Arkani-Hamed, N., Dimopoulos, S., and Dvali, G., Phys. Lett. B, 429, 263 (1998).

4. Randall, L., and Sundrum, R., Phys. Rev. Lett., 83, 3370 (1999).

5. Allanach, B. C., et al., Eur. Phys. J., C25, 113-123 (2002).

6. Baur, U., Phys. Rev. D, 42, 3 (1990).

7. Adloff, C., et al., Eur. Phys. J., C17, 567-581 (2000).

8. Heister, A., et al., Phys. Lett., B537, 5-20 (2002). 